\title{
Inhalt
}

\section{Briefwechsel 1802-1804}

*190. An Stephan August Winkelmann, vmtl. Anfang Januar 1802 . 3

191.P An Ludwig Wilhelm Gilbert, 2. Januar $1802 \ldots \ldots 3$

*192. Von ?, etwa 8. Januar $1802 \ldots \ldots \ldots \ldots$

193. Von Stephan August Winkelmann, etwa 11. Januar $1802 \ldots 4$

*194. Von Theodor Friedrich Arnold Kestner, etwa 11. Januar 180266

195. Von Clemens Brentano, 11. Januar $1802 \ldots \ldots . \ldots 6$

196. Von Johanna (Jeannette) Dieterich, 15. Januar $1802 \ldots . . . .8$

*197. An Theodor Friedrich Arnold Kestner, etwa 21. Januar 1802 . 9

198. An Clemens Brentano, 21. Januar 1802 ............ 9

*199. An Caroline von Labes, 21. Januar $1802 \ldots . . . . . . . . .11$

*200. An Caroline von Labes, 23. oder 27. Januar $1802 \ldots \ldots 12$

201. An Stephan August Winkelmann, 26. Januar $1802 \ldots . . . .12$

201.E An Stephan August Winkelmann, 26. Januar 1802 . . . . . . 17

202. Von Theodor Friedrich Arnold Kestner, 30. Januar 1802 . . . 19

203. Von Heinrich Dieterich, 1. Februar $1802 \ldots \ldots 21$

204. Von Caroline von Labes an Arnim und Carl Otto von Arnim, 2.-6. Februar $1802 \ldots \ldots \ldots . \ldots \ldots . \ldots \ldots 22$

205. Von Hans von Schlitz an Arnim und Carl Otto von Arnim, 6. und 7. Februar $1802 \ldots \ldots \ldots \ldots 25$

206. Von Clemens Brentano, vmtl. 8. Februar $1802 \ldots . . \ldots . .26$

207. Von Hans von Schlitz an Arnim und Carl Otto von Arnim, 15. Februar $1802 \ldots \ldots \ldots \ldots \ldots \ldots \ldots$. . . . . . . . . . . .

*208. Von Caroline von Labes an Arnim und Carl Otto von Arnim, vmtl. zwischen Mitte Februar und zweitem Drittel August 1802

*209. Von Caroline von Labes an Arnim und Carl Otto von Arnim, vmtl. zwischen Mitte Februar und zweitem Drittel August 1802 
Inhalt

*210. Von Caroline von Labes an Arnim und Carl Otto von Arnim, vmtl. zwischen Mitte Februar und zweitem Drittel August 1802 .............................

*211. Von Caroline von Labes an Arnim und Carl Otto von Arnim, vmtl. zwischen Mitte Februar und zweitem Drittel August 1802

*212. Von Caroline von Labes an Arnim und Carl Otto von Arnim, vmtl. zwischen Mitte Februar und zweitem Drittel August

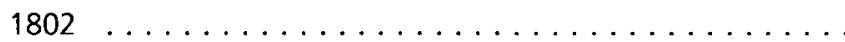

*213. Von Caroline von Labes an Arnim und Carl Otto von Arnim, vmtl. zwischen Mitte Februar und zweitem Drittel August 1802

*214. Von Caroline von Labes an Arnim und Carl Otto von Arnim, vmtl. zwischen Mitte Februar und zweitem Drittel August 1802

*215. Von Caroline von Labes an Arnim und Carl Otto von Arnim, vmtl. zwischen Mitte Februar und zweitem Drittel August

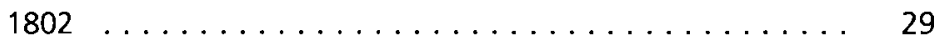

*216. An Hans von Schlitz, 17. Februar $1802 \ldots \ldots \ldots \ldots \ldots . . .30$

217. Von Stephan August Winkelmann, vmtl. etwa 20. Februar 1802

218. Von Hans von Schlitz an Arnim und Carl Otto von Arnim, 25. Februar - 1. März $1802 \ldots \ldots \ldots \ldots \ldots \ldots \ldots$. 31

219. An Clemens Brentano, 6., 7. und 15. März $1802 \ldots \ldots .32$

*220. An Hans von Schlitz, etwa 11. März $1802 \ldots \ldots \ldots \ldots$.

221. Von Hans von Schlitz, 14. März $1802 \ldots \ldots \ldots \ldots \ldots .34$

222. Von Hans von Schlitz, 15. März $1802 \ldots \ldots \ldots \ldots \ldots \ldots$.

*223. An Stephan August Winkelmann, vmtl. zwischen 16. und

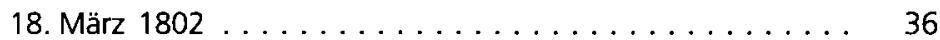

224. An Heinrich Dieterich, 16. März $1802 \ldots \ldots \ldots \ldots \ldots .36$

225. Von Louise von Schlitz an Arnim und Carl Otto von Arnim, 25. März $1802 \ldots \ldots \ldots \ldots \ldots \ldots \ldots \ldots \ldots \ldots$

*226. An Louise von Schlitz, vmtl. Ende März/Anfang April 1802 . . 40

227. Von Louise von Schlitz, 13. April $1802 \ldots \ldots \ldots \ldots \ldots .40$

228. Von Hans von Schlitz an Arnim und Carl Otto von Arnim,

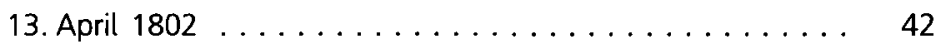

229.K An Clemens Brentano, 17. April 1802 ............ 43

229. An Clemens Brentano, 17. April $1802 \ldots \ldots \ldots \ldots . .44$ 
230.K An Clemens Brentano, 4. Mai $1802 \ldots \ldots . . . \ldots . . .46$

*230. An Clemens Brentano, 4. Mai $1802 \ldots . . \ldots$. . . . . . . 47

231. Von Clemens Brentano, vmtl. zwischen 10. und 12. Mai $1802 \quad 47$

*232. Von Clemens Brentano, vmtl. 20.-23. Mai $1802 \ldots 50$

233. Von Louise von Schlitz, 27. Mai $1802 \ldots \ldots \ldots$. . . . . . 50

*233a. An Johanna (Jeannette) Dieterich, vmtl. um den 20. Juni 180252

*234. An Louise von Schlitz, vmtl. zwischen Anfang und Mitte Juli

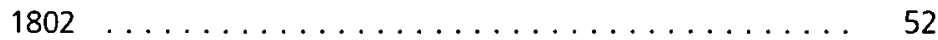

234.E An Louise von Schlitz, vmtl. zwischen Anfang und Mitte Juli

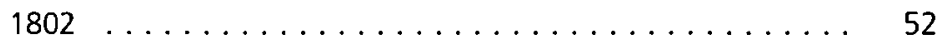

*235. Von Clemens Brentano, vmtl. zwischen Anfang und Mitte Juli

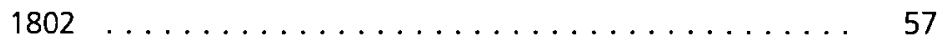

236.K An Clemens Brentano, 9. Juli $1802 \ldots \ldots \ldots 7$

236. An Clemens Brentano, 9. Juli $1802 \ldots \ldots 60$

*237. Von Caroline von Labes an Arnim und Carl Otto von Arnim, vmtl. zwischen Mitte Juli und Mitte August $1802 \ldots \ldots \ldots 66$

238. Von Louise von Schlitz, 27. Juli $1802 \ldots \ldots$. . . . . . . . 67

239. Von Louise von Schlitz, 28. Juli $1802 \ldots \ldots$. . . . . . . . 69

240. Von Clemens Brentano, vmtl. zwischen 3. und 7. August 180270

241.K An Clemens Brentano, vmtl. Beginn des zweiten Drittels August

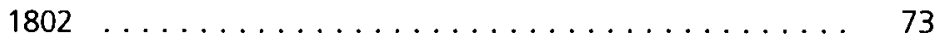

241. An Clemens Brentano, vmtl. Beginn des zweiten Drittels August

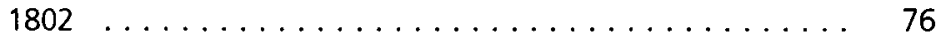

^242. An Stephan August Winkelmann, vmtl. Beginn des zweiten Drittels August $1802 \ldots \ldots \ldots \ldots \ldots \ldots$

*243. An Hans von Schlitz, vmtl. zweites Drittel August $1802 \ldots 81$

*244. Von Hans von Schlitz an Arnim und Carl Otto von Arnim, vmtl. zweites oder letztes Drittel August $1802 \ldots \ldots \ldots \ldots$. . . . 82

*245. Von Hans von Schlitz an Arnim und Carl Otto von Arnim, vmtl. zweites oder letztes Drittel August $1802 \ldots \ldots \ldots$. . . . 82

*246. An Caroline von Labes, vmtl. Mitte August $1802 \ldots . . . . .82$

247. An Heinrich Dieterich, 17. August $1802 \ldots \ldots . \ldots 2$

248.K An Louise von Schlitz, 17. August/1. September 1802 . . . . . 83

*249. An Johann Friedrich Zöllner, vmtl. zwischen Mitte August und Anfang September 1802 . . . . . . . . . . . . . . . . . . 84

250. Von Caroline von Labes an Arnim und Carl Otto von Arnim, 21.-30. August $1802 \ldots \ldots \ldots \ldots \ldots$

251. Von Clemens Brentano, 6. September $1802 \ldots \ldots$. . . . . 87 
252. Von Clemens Brentano, 8. September $1802 \ldots \ldots \ldots \ldots .91$

253.K1 An Clemens Brentano, zwischen 10. und 14. September 1802100

253.K2 An Clemens Brentano, zwischen 10. und 14. September 1802101

253.K3 An Clemens Brentano, zwischen 10. und 14. September 1802101

253.K4 An Clemens Brentano, etwa 22. September $1802 \ldots \ldots \ldots .103$

253. An Clemens Brentano, 14.-23. und 22. September 1802 ... 104

254. Von Hans von Schlitz an Arnim und Carl Otto von Arnim, 14. und 16. September 1802 . . . . . . . . . . . . . 117

*255. An Theodor Friedrich Arnold Kestner, vmtl. letztes Drittel September 1802 ........................ 119

*256. An Caroline von Labes, 20. September $1802 \ldots \ldots \ldots \ldots .119$

*257. Von Caroline von Labes an Arnim und Carl Otto von Arnim, 24. September $1802 \ldots \ldots \ldots \ldots \ldots \ldots \ldots \ldots \ldots . . \ldots 119$

*258. An Hans von Schlitz, vmtl. zwischen Ende September und Ende Oktober 1802 . . . . . . . . . . . . . . . . . . 120

259. Von Bettina, Kunigunde und Clemens Brentano, 7. Oktober 1802 .............................. 120

260. Von Clemens Brentano, 7./8. Oktober $1802 \ldots \ldots \ldots \ldots .121$

*261. Von Stephan August Winkelmann, vmtl. 8. Oktober 1802 ... 130

*262. An Caroline von Labes, 11. Oktober $1802 \ldots . \ldots . . .130$

263. Von Theodor Friedrich Arnold Kestner, vmtl. zweite Hälfte Oktober 1802 ......................... 130

264. Von Caroline von Labes an Arnim und Carl Otto von Arnim, 16. Oktober 1802 ...................... 133

*265. Von Caroline von Labes, 30. Oktober $1802 \ldots \ldots \ldots \ldots .134$

266. Von Hans von Schlitz an Arnim und Carl Otto von Arnim, 7. November 1802 . . . . . . . . . . . . . . . . . . . 135

267. An Stephan August Winkelmann, 8. November $1802 \ldots \ldots .137$

*268. An Christian Leopold von Buch, zwischen 9. und 19. November 1802 . . . . . . . . . . . . . . . . . . . . . . . . 139

*269. An Louise von Schlitz, vmtl. zwischen Mitte und 18. November 1802 ... . . . . . . . . . . . . . . . . . . . . . . . 139

270. Von Hans von Schlitz an Arnim und Carl Otto von Arnim, 17. November 1802 . . . . . . . . . . . . . . . . . . . 140

271. An Clemens Brentano, 18. November $1802 \ldots \ldots . . . .140$

271.E An Clemens Brentano, 18. November $1802 \ldots \ldots \ldots \ldots .148$

272. An Bettina und Kunigunde Brentano, 18. November 1802 .. 150

*273. An Caroline von Labes, 18. November $1802 \ldots \ldots \ldots . .152$ 
*274. An Gebrüder Bethmann, 19. November $1802 \ldots \ldots$. . . 152

275. Von Louise von Schlitz, letztes Drittel November 1802 bis Januar $1803 \ldots \ldots \ldots \ldots \ldots \ldots \ldots \ldots \ldots . \ldots \ldots 2$

*276. Von Caroline von Labes, 23. November 1802 . . . . . . . 155

277. Von Caroline von Labes an Arnim und Carl Otto von Arnim, 30. November $1802 \ldots \ldots \ldots \ldots \ldots$. . . . . . . . . . 155

278. Von Gebrüder Bethmann, 4. Dezember $1802 \ldots \ldots \ldots \ldots 157$

279. Von Clemens Brentano, etwa Mitte bis etwa 18. Dezember $1802 \ldots \ldots \ldots \ldots \ldots \ldots \ldots \ldots \ldots \ldots \ldots \ldots . \ldots \ldots$

280. An Hans von Schlitz, 30. Dezember $1802 \ldots \ldots \ldots \ldots .166$

280.E An Hans von Schlitz, 30. Dezember $1802 \ldots \ldots \ldots \ldots \ldots 168$

281.K1 An Clemens Brentano, 12. bis letztes Drittel Januar 1803 ... 169

281.K2 An Clemens Brentano, 12. bis letztes Drittel Januar $1803 \ldots 170$

281. An Clemens Brentano, 12. bis letztes Drittel Januar 1803 ... 173

*282. An Heinrich Dieterich, zwischen letztem Drittel Januar und Anfang April $1803 \ldots \ldots \ldots \ldots \ldots \ldots$. . . . . . . 183

*283. An Johanna (Jeannette) Dieterich, zwischen letztem Drittel Januar und Anfang April $1803 \ldots \ldots \ldots \ldots \ldots \ldots$

284. Von Johann Friedrich Reichardt, zwischen letztem Drittel Januar

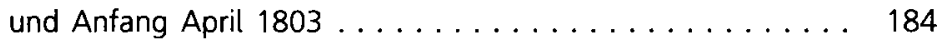

285. Von Helmina von Hastfer (Chézy), zwischen letztem Drittel Januar und Mitte Juni $1803 \ldots \ldots \ldots \ldots$. . . . . . . . . 184

286. Von Helmina von Hastfer (Chézy), zwischen letztem Drittel Januar und Mitte Juni 1803 . . . . . . . . . . . . . . . 185

287. Von Clemens Brentano, Mitte Februar $1803 \ldots \ldots \ldots \ldots .185$

288.K1 An Clemens Brentano, 17. Februar 1803 . . . . . . . . . 193

288.K2 An Clemens Brentano, vmtl. Anfang März 1803 . . . . . . 195

288.K3 An Clemens Brentano, zwischen 17. Februar und 1. März 1803 ... . . . . . . . . . . . . . . . . . . . . 199

288. An Clemens Brentano, 17. Februar, 1. und 7. März 1803 ... 200

288.E An Clemens Brentano, 7. März $1803 \ldots \ldots \ldots \ldots \ldots .208$

289. An Hans von Schlitz, 21. Februar $1803 \ldots \ldots \ldots \ldots . \ldots .209$

290.K An Clemens Brentano, 4./5. April 1803 . . . . . . . . . . 210

*290. An Clemens Brentano, 4.-12. April 1803 ........... 211

291. Von Anne Louise Germaine de Staël-Holstein, 12. April 1803211

*292. An Johann Ludwig Formey, vmtt. Mitte April 1803 . . . . . . 212

293. An Friedrich Carl von Savigny, 17. April $1803 \ldots \ldots \ldots .213$

294. Von Friedrich Carl von Savigny, 27. April 1803 ........ 214 
295. Von Clemens Brentano (mit Teilabschrift eines Briefes von Gustav Ludwig Johann von Wrangel), 30. April $1803 \ldots . .214$

296. Von Johann Ludwig Formey, 1. Mai $1803 \ldots \ldots \ldots \ldots \ldots .226$

297. Von Georg Ludwig Spalding, 3. Mai $1803 \ldots \ldots \ldots . . . .227$

298. An Clemens Brentano, 5. Mai 1803 . . . . . . . . . . . 229

298.E An Clemens Brentano, 5. Mai $1803 \ldots \ldots \ldots \ldots \ldots .234$

*299. An Joachim Erdmann von Arnim, 5. Mai $1803 \ldots \ldots$. . . . 236

300.K An Stephan August Winkelmann, zwischen 5. und Mitte Mai

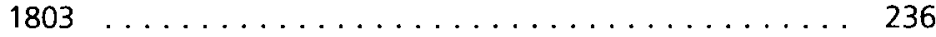

301. Von Johanna (Jeannette) Dieterich, 6. Mai $1803 \ldots \ldots . . .239$

302. Von Clemens Brentano, 11. Mai $1803 \ldots \ldots \ldots \ldots \ldots .240$

303. Von Clemens Brentano, 11. Mai 1803 ............. 241

304.K An Anne Louise Germaine de Staël-Holstein, 13. Mai 1803 . . 245

304. An Anne Louise Germaine de Staël-Holstein, 14. Mai 1803 . . 247

305. Von Helmina von Hastfer (Chézy), vmtl. zwischen Mitte Mai und Mitte Juni 1803 . . . . . . . . . . . . . . . . . . . . . . . 249

306.K An Helmina von Hastfer (Chézy), vmtl. zwischen Mitte und 24. Mai 1803 . . . . . . . . . . . . . . . . . . . . . . . . 250

*306. An Helmina von Hastfer (Chézy), vmtl. zwischen Mitte und 24. Mai $1803 \ldots \ldots \ldots \ldots \ldots \ldots \ldots . \ldots \ldots \ldots 252$

307. Von Helmina von Hastfer (Chézy), 24. Mai $1803 \ldots \ldots \ldots .253$

308.K An Helmina von Hastfer (Chézy), 24. Mai 1803 . . . . . . . 254

309. Von Joachim Erdmann von Arnim, 25. Mai $1803 \ldots \ldots \ldots .255$

310. An Clemens Brentano, 6. Juni $1803 \ldots \ldots \ldots \ldots \ldots .256$

311. Von Helmina von Hastfer (Chézy), 14. Juni $1803 \ldots \ldots . .261$

312. An Helmina von Hastfer (Chézy), etwa 18. Juni 1803 ..... 262

*313. Von Caroline von Labes, vmtl. Juli $1803 \ldots \ldots \ldots \ldots \ldots .263$

314. An Clemens Brentano, 5. Juli 1803 . . . . . . . . . . . . 263

314.E1 An Clemens Brentano, 5. Juli $1803 \ldots \ldots \ldots \ldots \ldots .268$

314.E2 An Clemens Brentano, 5. Juli 1803 . . . . . . . . . . . 270

315. An Helmina von Hastfer (Chézy), 7. August 1803 ...... 271

*316. An Dorothea Veit und vmtl. Friedrich Schlegel, vmtl. 7. August $1803 \ldots \ldots \ldots \ldots \ldots \ldots \ldots \ldots \ldots \ldots . \ldots \ldots 272$

317.K An Louise von Schlitz, vmtl. zweites Drittel August 1803 .. 273

318.K1 An Clemens Brentano (?), zweites Drittel August $1803 \ldots .275$

318.K2 An Clemens Brentano, zweites Drittel August $1803 \ldots \ldots .277$

318. An Clemens Brentano, 19. August 1803 . . . . . . . . 277

319. Von Clemens Brentano, vmtl. zwischen 17. und 23. August $1803 \ldots \ldots \ldots \ldots \ldots \ldots \ldots \ldots \ldots \ldots \ldots . \ldots \ldots$ 
Inhalt

320.K An ?, 19. August $1803 \ldots \ldots \ldots \ldots$. . . . . . . . . 295

*321. Von Helmina von Hastfer (Chézy), Dorothea Veit und vmtl.

Friedrich Schlegel, vmtl. zwischen Anfang September und Ende Dezember 1803 . . . . . . . . . . . . . . . . . . . . . . . . . . 295

322.K1 An Ludwig Wilhelm Gilbert, Oktober oder November 1803 . . 295

322.K2 An Ludwig Wilhelm Gilbert, Oktober oder November 1803 . . 296

323. Von Clemens Brentano, 12. Oktober 1803 . . . . . . . . 297

324.K An Hans von Schlitz (?), vmtl. November oder erste Hälfte Dezember 1803 . . . . . . . . . . . . . . . . . . . . . 309

*325. An Helmina von Hastfer (Chézy), Dorothea Veit und vmtl. Friedrich Schlegel, vmtl. zwischen Mitte Dezember 1803 und Mitte März 1804 . . . . . . . . . . . . . . . . . . . . . . 309

*326. An Johanna (Jeannette) Dieterich, vmtl. zweite Hälfte Dezember $1803 \ldots \ldots \ldots \ldots$. . . . . . . . . . . . . . . 310

327.K1 An Clemens Brentano, etwa 24. Dezember 1803 . . . . . . 310

327.K2 An Clemens Brentano, etwa 24. Dezember 1803 . . . . . . 310

327. An Clemens Brentano, 24., 26. und 27. Dezember 1803 . . 312

327.E An Clemens Brentano, 27. Dezember 1803 . . . . . . . . . 340

*328. Von Caroline von Labes, vmtl. zwischen letztem Drittel Januar und Ende Februar $1804 \ldots \ldots \ldots \ldots$. . . . . . . . . 342

*329. Von Hans von Schlitz, vmtl. zwischen letztem Drittel Januar und Ende Februar $1804 \ldots$. . . . . . . . . . . . . . . . . . . . . 342

330. Von Johanna (Jeannette) Dieterich, 23. Januar $1804 \ldots 343$

331.K An Johanna (Jeannette) Dieterich, 9. Februar 1804 . . . . . . 344

*332. An Caroline von Labes, 1. März $1804 \ldots \ldots$. . . . . . . . . 345

333. Von Clemens Brentano, 1. März $1804 \ldots \ldots$. . . . . . . . 345

334.K An Clemens Brentano, 2. März 1804 . . . . . . . . . . . . . . 347

334. An Clemens Brentano, 2. März $1804 \ldots . . . . . . . . . .349$

335.K An Hans von Schlitz, 6. März $1804 \ldots \ldots 51$

336.K1 An Clemens Brentano, vmtl. zwischen Ende März und Ende April $1804 \ldots \ldots \ldots \ldots \ldots$. . . . . . . . . . . 353

336.K2 An Clemens Brentano, vmtl. zwischen Ende März und Ende April $1804 \ldots \ldots \ldots$. . . . . . . . . . . . . . . 357

336.K3 An Clemens Brentano, vmtl. zwischen Ende März und Ende April $1804 \ldots \ldots$. . . . . . . . . . . . . . . . . . 362

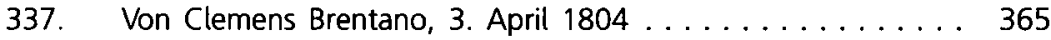

338. Von Helmina von Hastfer (Chézy), Dorothea Veit und Friedrich Schlegel, 4. April $1804 \ldots \ldots$. . . . . . . . . . . . . . 368 
339. Von Caroline von Labes an Arnim und Carl Otto von Arnim, 7. April $1804 \ldots \ldots \ldots \ldots \ldots \ldots \ldots \ldots \ldots \ldots . \ldots \ldots$

340.K An Clemens Brentano, vmtl. Ende April/Anfang Mai 1804 .. 372

340. An Clemens Brentano, vmtl. Ende AprivAnfang Mai 1804 ... 373

341.K An Barbara Juliane von Krüdener, 1. Mai $1804 \ldots \ldots . . .376$

342.K An Hans von Schlitz, 18. Mai $1804 \ldots \ldots \ldots \ldots . \ldots . . .6377$

343. Von Clemens Brentano, 23. Mai $1804 \ldots \ldots \ldots \ldots \ldots . . \ldots 378$

344.K An Clemens Brentano, 12. August $1804 \ldots \ldots \ldots \ldots . . .379$

344. An Clemens Brentano, 12. August $1804 \ldots \ldots \ldots \ldots . .380$

*345. An Theodor Friedrich Arnold Kestner, vmtl. zwischen Mitte August und Ende Oktober 1804 . . . . . . . . . . . . . 381

346. Von Clemens Brentano, 28. August $1804 \ldots \ldots . \ldots . . . . .381$

347. An Heinrich Dieterich, zwischen Anfang und 19. September

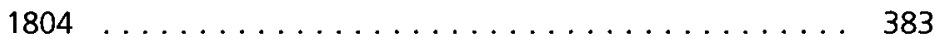

348. An Clemens Brentano, 20. September $1804 \ldots \ldots \ldots . .383$

349. Von Clemens Brentano, 3. Oktober $1804 \ldots \ldots \ldots \ldots . .385$

350. An Clemens Brentano, 3. Oktober $1804 \ldots \ldots \ldots \ldots \ldots .388$

351. Von Clemens Brentano, 25. Oktober $1804 \ldots \ldots . \ldots . .390$

352. An Clemens Brentano, vmtl. Anfang November $1804 \ldots . . .392$

353. An Sara Levy, vmtl. Anfang November 1804 ... . . . . . . 393

*354. An Friedrich Tieck, zwischen Anfang November und Mitte Dezember 1804 ... . . . . . . . . . . . . . . . . . . . . . . . 393

355. Von Theodor Friedrich Arnold Kestner, 13. Dezember 1804 . . 393

*356. Von Friedrich Tieck, vmtl. zwischen letztem Drittel Dezember 1804 und Ende Januar 1805 . . . . . . . . . . . . . . . . 395

357. Von Caroline von Labes, 24. Dezember $1804 \ldots . . . . . . .395$

358. Von Clemens Brentano, 26. Dezember $1804 \ldots \ldots \ldots \ldots .396$

*359. An Friedrich Tieck, vmtl. zwischen Ende Dezember 1804 und Mitte Februar 1805 . . . . . . . . . . . . . . . . . . . . . 398 
Inhalt

\section{Anhang 1}

Eintragungen in Arnims Stammbuch und ein Fremdenbuch (1801-1804)

Al.47. Arnim und Carl Otto von Arnim, 26. November 1801 . . . . 401

Al.48. Helmina von Hastfer (Chézy), vmtl. zweite Hälfte Mai 1803 . 401

Al.49. J. M. von Fellner, vmtl. zwischen Anfang Oktober und Mitte Dezember 1803 . . . . . . . . . . . . . . . . . . . . . . . . . 401

Al.50. Clemens Brentano, 18. Dezember $1804 \ldots \ldots$. . . . . . . . 401

\section{Anhang II}

Kontextbriefe (1802-1803)

All.13. Clemens Brentano an Friedrich Carl von Savigny, 30. Juni/ 1. Juli $1802 \ldots \ldots \ldots \ldots$. . . . . . . . . . . . . . . . 405

All.14. Louise von Schlitz an Clemens Brentano, 28. Juli 1802 . . . . 405

All.15. Juliette von Krüdener an Camille Jordan, 19. Januar 1803 . . 407

\section{Kommentar}

Zu dieser Ausgabe . . . . . . . . . . . . . . . . . . . . . . . . 411

Editorische Abkürzungen und Zeichen . . . . . . . . . . . . 421

Abkürzungen und Zeichen in den Texten . . . . . . . . . . . . . . 424

Abgekürzt zitierte Literatur . . . . . . . . . . . . . . 426

Zum Briefwechsel . . . . . . . . . . . . . . . . . . . . . 445

Zu Anhang I . . . . . . . . . . . . . . . . . . . 923

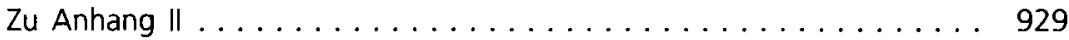

Gedichtanfänge und -überschriften . . . . . . . . . . . . . . 935

Korrespondenten und Zeugen . . . . . . . . . . . . . . . . 941

Personenregister . . . . . . . . . . . . . . . . . . 961 
